\title{
Injectable Astragalus membranaceus Polysaccharide
}

National Cancer Institute

\section{Source}

National Cancer Institute. Injectable Astragalus membranaceus Polysaccharide. NCI

Thesaurus. Code C103822.

An injectable form of a polysaccharide isolated from the radix of Astrag alus

membranaceus (PG2), used in traditional Chinese medicine, with potential hematopoiesis inducing and immunomodulating activities. Upon injection, APS may be able to relieve certain chemotherapy-induced side effects, including myelosuppression, fatigue, mucositis, pain, nausea and vomiting, as well as loss of appetite and body weight. Also, APS may exert immunostimulatory activities by stimulating B-lymphocytes, activating Tlymphocytes, inducing cytokine production, and activating macrophages and natural killer cells through as of yet unidentified mechanism(s). APS may improve compliance of radiotherapy and/or chemotherapy. 